\title{
Challenges of fully-coupled high-fidelity ditching simulations
}

\author{
Maximilian Müller ${ }^{1,}{ }^{*}$, Malte Woidt ${ }^{1}$, Matthias Haupt ${ }^{1}$, Peter Horst ${ }^{1}$ \\ ${ }^{1}$ TU Braunschweig, Institute of Aircraft Design and Lightweight Structures, Hermann-Blenk-Str. \\ 35, D-38108 Braunschweig
}

\begin{abstract}
An important element of the process of aircraft certification is the demonstration of the crashworthiness of the structure in the event of an emergency landing on water, also referred to as ditching. Novel numerical simulation methods that incorporate the interaction between fluid and structure open up a promising way to model ditching in full scale. This study presents a numerical framework for the simulation of ditching on a highfidelity level. A partitioned approach that combines a finite volume hydrodynamic fluid solver as well as an finite element structural solver is implemented using a Python-based distributed coupling environment [1]. High demands are placed both on the fluid and the structural solver. The fluid solver needs to account for hydrodynamic effects such as cavitation in order to correctly compute the ditching loads acting on the aircraft structure. In the structural model, the highly localized damage induces nonlinearities and large differences in model scale. In order to reduce the computational effort a reduced order model is used to model the failure of fuselage frames. The fluid-structure coupling requires an explicit coupling scheme. It is shown that the standard Dirichlet-Neumann approach exhibits unstable behaviour if a strong added-mass effect is present, as is the case in aircraft ditching. This indicates a need for methods other than the standard DirichletNeumann approach [2].
\end{abstract}

\section{Introduction}

Commercial transport aircraft often fly above open waters and have to prove the safe landing on water, also referred to as ditching. Therefore, ditching is part of the certification specifications by EASA and FAA. Those regulations aim for minimizing the risk of injury of the occupants of the aircraft. This requires an accurate prediction of the structural behaviour of the fuselage during the impact phase of ditching. Furthermore, the degree of damage and its influence on the floatation capabilities of the structure need to be determined to allow the safe evacuation of the aircraft during the floatation phase.

Table 1. Overview of challenges of high-fidelity ditching simulations

\begin{tabular}{|l|l|l|}
\hline \multicolumn{1}{|c|}{$\begin{array}{c}\text { Structural } \\
\text { simulation }\end{array}$} & \multicolumn{1}{c|}{$\begin{array}{c}\text { Fluid } \\
\text { simulation }\end{array}$} & \multicolumn{1}{c|}{$\begin{array}{c}\text { Interaction between } \\
\text { fluid and structure }\end{array}$} \\
\hline $\begin{array}{l}\text { - highly localized damage } \\
\text { - large differences in model } \\
\text { scale }\end{array}$ & $\begin{array}{l}\text { - multiphase flow } \\
- \text { hydrodynamic effects, e.g. } \\
\text { cavitation and ventilation } \\
\text { (see e.g. [3]) }\end{array}$ & $\begin{array}{l}\text { - incorporation of explicit struc- } \\
\text { tural solver in partitioned } \\
\text { coupling approach } \\
\text { instability of coupling due to } \\
\text { strong added mass effect }\end{array}$ \\
\hline
\end{tabular}

* Corresponding author: maximilian.mueller@tu-braunschweig.de 


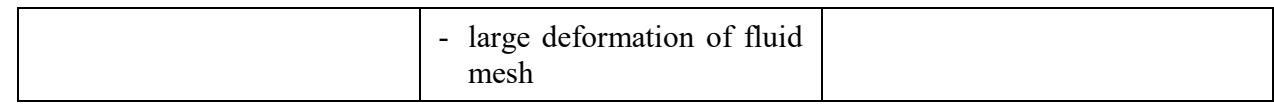

Simulating ditching needs a full-scale approach to accurately account for the various physical phenomena that are relevant for ditching (see e.g. [3]). As shown by Reinhard et al. in [4], the flexibility of the structure needs to be considered as it has a significant effect on the hydrodynamic loads acting on the structure and therefore on the trajectory of the aircraft. On that account, it is essential to account for fluid-structure interaction (FSI). Different numerical challenges of high-fidelity ditching simulations as well as relevant physical phenomena are summarized in Table 1 . The high-fidelity approach presented in this study can serve different purposes, such as replacing expensive model tests or training lowerfidelity models that are used in the design process of the aircraft.

The remainder of this paper is structured as follows. In the next section, we introduce the numerical framework as well as the implicit coupling approach used in this study. In section 3 , the framework is validated by means of two simple model problems. The first model problem describes the drop test of a rigid cylinder. In the second validation case the model problem is extended to account for the elasticity of the cylinder. This case will be simulated using both an implicit and an explicit time integration scheme in the structural solver. Section 4 deals with the development of a structural model of the aircraft fuselage. The particular challenge is to use a sufficient fine mesh in the impacting region while limiting the computational effort to a manageable amount. This is achieved by reducing the order of the model. A summary of the work and some conclusions are given in section 5 .

\section{Numerical Framework}

The coupling environment ifls used in this study allows to couple arbitrary black-box solvers in a partitioned way (see e.g. [5]). The architecture of the framework is shown in Fig. 1a. The control code allows the implementation of different coupling methods such as iterative or simple staggered methods. The communication and data exchange with different solvers is achieved through Python-based co-processes. The interpolation of coupling values between different surface meshes at the FSI interface is realized by VTK-based interpolation filters.

For the simulation of the fluid domain, the finite-volume interDyMFoam solver which is part of the OpenFOAM 5 toolbox is employed. This high-fidelity solver solves the incompressible Navier-Stokes equations and is able to model multiphase flow by applying the volume of fluid (VOF) method. The fluid equations are solved in the Arbitrary Lagrangian-Eulerian (ALE) form to account for the movement of the fluid mesh in the convective term. The fluid solver is coupled to ifls using a socket-based data exchange. The structure is simulated with the implicit Abaqus solver where linear elastic material behaviour is assumed.

The general partitioned FSI problem can be formulated as follows. The whole computational domain $\Omega$ is divided into the structural domain $\Omega_{S}$ and the fluid domain $\Omega_{F}$ with their respective boundaries $\partial \Omega_{\mathrm{S}}$ and $\partial \Omega_{\mathrm{F}}$. Both domains share a common boundary $\Gamma_{\mathrm{i}} \stackrel{\text { def }}{=} \partial \Omega_{\mathrm{S}} \cap \partial \Omega_{\mathrm{F}}$, referred to as the FSI interface. At $\Gamma_{\mathrm{i}}$ the coupling values are exchanged through appropriate boundary conditions. In the fluid field, the Dirichlet boundary condition

$$
\boldsymbol{v}_{f}=\boldsymbol{v}_{\boldsymbol{s}}, \quad \text { on } \Gamma_{\mathrm{i}}
$$

is applied, where $v_{f}$ is the fluid velocity vector and $v_{s}$ the velocity vector of the structure. Equation (1) can be considered a no-slip condition on the FSI interface.

On the structural side, the fluid forces are applied as the Neumann boundary condition

$$
\boldsymbol{\sigma}_{\boldsymbol{f}} \boldsymbol{n}=\boldsymbol{\sigma}_{\boldsymbol{s}} \boldsymbol{n}, \text { on } \Gamma_{\mathrm{i}}
$$


where $\sigma_{f}$ and $\boldsymbol{\sigma}_{\boldsymbol{s}}$ represent the fluid and structural stress tensor, respectively, and $\boldsymbol{n}$ stands for the unit normal vector.

Due to the relatively high ratio between fluid and structural density, ditching entails a pronounced added-mass effect. As stated by Causin et al. in [6] this can lead to instability of the coupled simulation or increased computational effort if the classical Dirichlet-Neumann approach is used. For that reason, the implicit coupling procedure illustrated in Fig. 1b, which is usually stable for strongly coupled problems, is employed. This procedure requires several equilibrium iterations per time step until a displacement convergence criterion is reached.

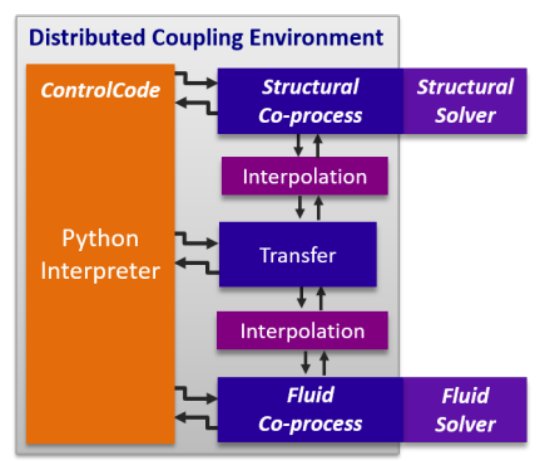

Fig. 1a. Distributed coupling environment ifls (see [5])

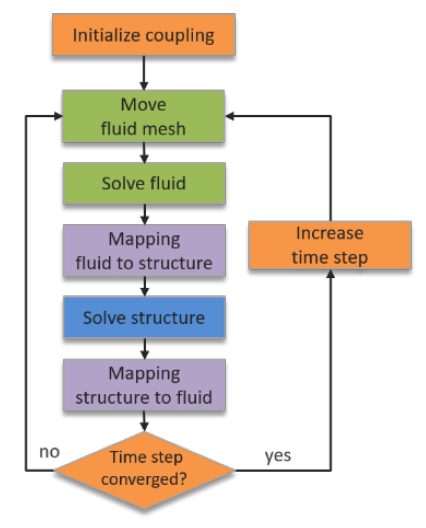

Fig. 1b. Implicit coupling procedure

\section{Validation studies}

\subsection{Drop test of rigid cylinder}

The model problem of the drop test of a rigid cylinder is based on the experimental study [7] by Greenhow and Lin. The problem set-up as well as the boundaries of the computational domains are shown in Fig. 2a. Table 2 summarizes the velocity and pressure boundary conditions that are valid in the fluid domain. At $\Gamma_{i}$, the interface conditions described in section 2 are applied. The implicit coupling scheme shown in Fig. $1 \mathrm{~b}$ is used.

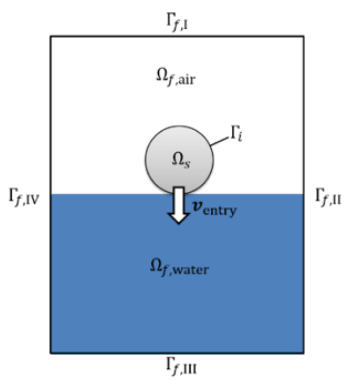

Fig. 2a. Schematic representation of drop test

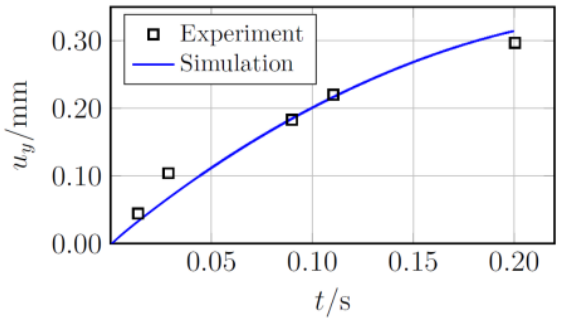

Fig. 2b. Results from drop test of rigid cylinder, experimental results from [7]

The diameter of the cylinder is set to $d=0.11 \mathrm{~m}$. The mass is chosen in such a way that the cylinder is neutrally-buoyant. The cylinder entries the water at a velocity of $\left|v_{\text {entry }}\right|=2.955 \mathrm{~m} / \mathrm{s}$.

Table 2. Boundary conditions in fluid domain

\begin{tabular}{|l|l|l|}
\hline Boundary & Pressure & Velocity \\
\hline
\end{tabular}




\begin{tabular}{|l|l|l|}
\hline$\Gamma_{f, \mathrm{I}}$ & $\begin{array}{l}\text { Constant zero pressure (atmos- } \\
\text { phere) }\end{array}$ & $\begin{array}{l}\text { Outflow: } \\
\text { Inflow: }\end{array}$ \\
$\begin{array}{l}\text { zero gradient in normal direction } \\
\text { Robin condition based on flux } \\
\text { in the patch-normal direction }\end{array}$ \\
$\Gamma_{f, \mathrm{II}-\mathrm{IV}}$ & Zero gradient in normal direction & Constant zero velocity \\
\hline
\end{tabular}

The fluid domain is spatially discretized by a two-dimensional O-Grid containing $91 \mathrm{k}$ cells. The solid is meshed with rigid surface elements where point masses are used to model the mass of the cylinder. Fig. $2 \mathrm{~b}$ shows the depth of penetration of the cylinder calculated by the simulation in comparison to the experimental results from [7]. The results show that the coupled simulation is able to reproduce the experimental results.

\subsection{Drop test of deformable cylinder}

The elastic validation case is based on [8] by Arai and Miyauchi. In their study, the impact of cylindrical shells on water is both experimentally and numerically investigated. The parameters of the second validation case are summarized in Table 3.

Table 3. Parameters of elastic validation case

\begin{tabular}{|c|c|}
\hline $\begin{array}{l}\text { Outer cylinder diameter } \\
\text { Shell thickness } \\
\text { Entry velocity } \\
\text { Shell Density } \\
\text { Young's modulus } \\
\text { Poisson's ratio }\end{array}$ & $\begin{aligned} d & =0.306 \mathrm{~m} \\
t & =3 \cdot 10^{3} \mathrm{~m} \\
\left|v_{\text {entry }}\right| & =4.429 \mathrm{~m} / \mathrm{s} \\
\rho_{s, 1} & =2.70 \cdot 10^{3} \mathrm{~kg} \mathrm{~m}^{-3} \\
\rho_{s, 2} & =3.04 \cdot 10^{3} \mathrm{~kg} \mathrm{~m}^{-3} \\
E_{s} & =7.35 \cdot 10^{10} \mathrm{~Pa} \\
v_{s} & =0.34\end{aligned}$ \\
\hline
\end{tabular}

The experimental results from [8] are influenced due to additional masses in the experimental setup (e.g. cables, connectors or sensors). Following the simplified approach by Ionina and Korobkin from [9], the shell density is increased by $11 \%$. In Table 3, $\rho_{s, 1}$ represents the density without additional mass, whereas $\rho_{s, 2}$ accounts for the additional mass.

The elastic shell is modelled by shell elements with linear form functions. Geometric non-linearities are accounted for. Despite different dimensions and the elasticity of the cylinder, the simulation model is equivalent to the model described in section 3.1.

Fig. 3a shows the numerical and experimental strain response at the bottom of the cylinder. The numerical results show good agreement in terms of the extreme values. Anyhow, the dependency on time is not reproduced properly. Only a small improvement is achieved if the additional mass in the experimental setup is considered. A possible reason for that observation is that, in reality, additional masses are not uniformly distributed in the cylinder as it is assumed here.

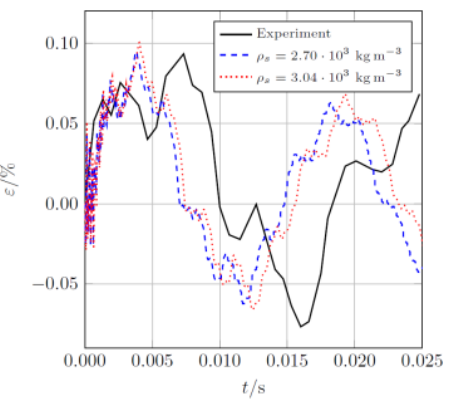

Fig. 3a. Strain results from drop test of deformable cylinder, experimental data from [8]

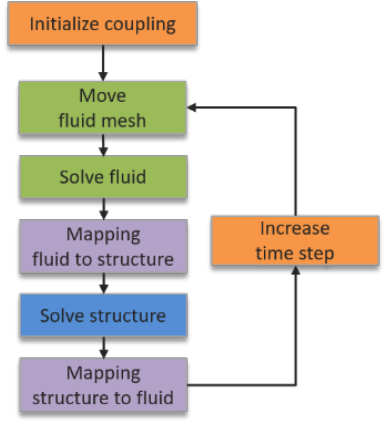

Fig. 3b. Explicit coupling procedure 


\subsection{Drop test of deformable cylinder using explicit structural Solver}

The simulation is conducted with two different coupling step sizes $\Delta t_{C P, 1}=4 \cdot 10^{-7} \mathrm{~s}$ and $\Delta t_{C P, 2}=1 \cdot 10^{-7} \mathrm{~s}$. Both step sizes result in unstable behaviour of the coupled simulation as soon as the cylinder comes into contact with the water. This shows that standard Dirichlet-Neumann boundary conditions are not suitable for strongly coupled problems if an explicit structural solver is coupled using an explicit coupling procedure (see Fig. 3b).

\section{Structural model}

\subsection{Global and component FEM-model}

Preliminary ditching simulations with a rigid aircraft of A350 size show, that an approx. $10 \mathrm{~m}$ long section of the rear fuselage is subjected to water loads during the impact phase of a ditching event. The section is shown in Fig.4. Still, the whole aircraft needs to be structurally modelled to capture the overall dynamics of the aircraft during impact. If structural damage occurs, it is expected to be in the green region.

An example for damage that is expected is breaking of a fuselage frame. A simulation of such an event due to bending moments acting on the frame is shown in Fig. 5b. The damage is highly localized and magnitudes of order smaller than the overall aircraft dimensions. Due to the complex damage mechanisms that are triggered by a stability buckling in the inner flange

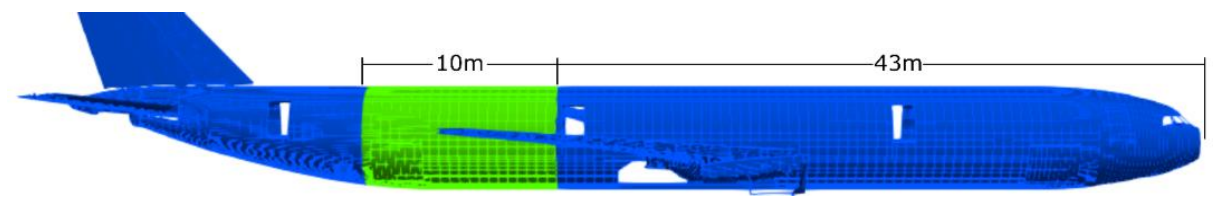

Fig 4. Ditching relevant region (highlighted in green) of an A350 sized Aircraft

or by plasticity effects, a very fine mesh is needed. A preliminary mesh size study of the frame failure under moment is presented in Fig.6, showing the bending angle over the applied momentum for different element edge length. Due to the high nonlinearities an explicit time integration is used for the structural simulation. As the smallest element of the model determines the time step size in explicit integration schemes a model that can directly account for damage and captures the hole aircraft is not a feasible approach for this kind of simulations as the expected simulated time for the impact phase of ditching is several seconds. Therefore, we propose to use a multiscale model for the structural part.

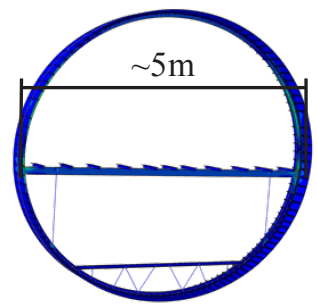

Fig. 5a. Slice of the mid-detailed FEM Model (green region)
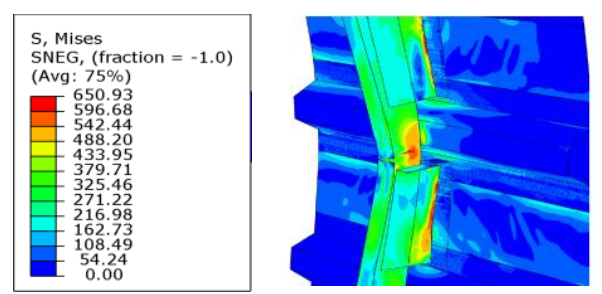

Fig. 5b. Damage FEM of a part of the Frame subjected to a bending moment 


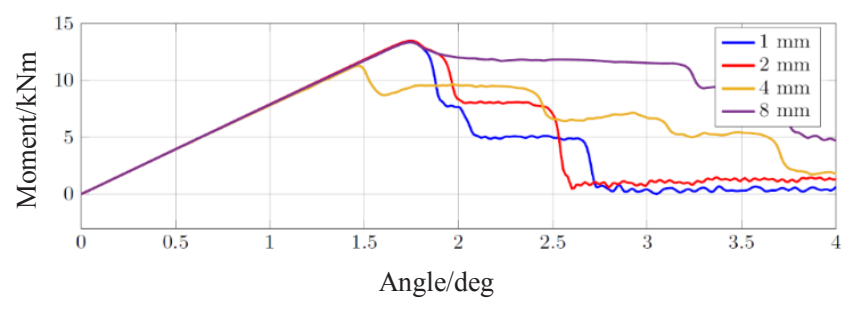

Fig. 6. Results of the Model in Fig. 5b with different element side length

\subsection{Multiscale model for ditching simulation}

To capture the overall aircraft dynamics outside the green region in Fig. 4 a "Global FEM Model" (GFEM) is used. Stringers and frames are modelled as rods and beams on a skin modelled as shells. The whole aircraft has approx. 300k degrees of freedom, masses are defined as point masses that are distributed to whole fuselage sections. The overall dynamics can be captured by such models that are widely used in industry.

The region highlighted in greed needs a much finer discretization. As the water loads are applied on the outside of the skin it is important to capture how this loads are transferred to the stiffening structure of the fuselage. Therefore, frames, stringers, floor beams and clips are all separately modelled as shells as shown in Fig. 5a. This gives better insights into the loading states of the distinct structural parts of the fuselage what cannot be achieved by GFEM models. Also a finer mass model is needed as inertia forces are important for the loading state during highly dynamic events as ditching. The floor struts are still modelled as beams as their loading state is mainly axial compression for what a beam discretization is sufficient. The mesh size is chosen to be $10-20 \mathrm{~mm}$ to limit the whole model size of the whole $10 \mathrm{~m}$ region. The resulting models have approx. 10M degrees of freedom and therefore can be handled by commercial explicit FEM solvers. The rest of the aircraft is taken form the GFEM model that is compared to the detailed model very small in size. The two models are combined by multipoint-constraints and simulated together. This leads to an aircraft model that is locally refined. This model is still too coarse to account for damage effects.

As stated earlier further local refinement of the model in very small parts that are subjected to damage would not dramatically increase the model size but would decrease the integration time step massively. The model would become unfeasible to simulate a physical time span of several seconds. Therefore, the damage is integrated into the model by reduced order models.

\subsection{Modelling Damage}

As seen in Fig. 3 the damage in aircraft structures is highly localized. Most parts of structure are only subjected to characteristic loadings. The stringers together with the skin are for example mostly subjected to longitudinal stresses introduced by bending of the fuselage, the frames are mainly loaded by bending moments and hoop stresses to keep the fuselage crosssections in shape and the clips bear mainly shear forces to transfer the loads from the skin to the frame. These assumptions make it possible to easily introduce reduced order models that can simulation damage effects in relatively coarse FEM models. The approach is called "Kinematics Model" and is described in [10]. For instance, the damage of a frame can be modelled as a non-linear hinge in the frame with the rotation point at the skin. The hinge is closed for uncritical loads and starts to open at a certain point at which the frame starts to break. The moment to opening angle curve is obtained by a detailed FEM simulation. As the damage influence region is very small the overall effect of the damage can be captured by such approaches with sufficient accuracy. 


\subsection{Creating the multiscale model}

To create a reduced order damage model it is necessary to first identify locations of the model where a damage might occur during ditching and to identify the characteristic loading of the relevant parts. To do this an iterative process is proposed as shown in Fig. 7.

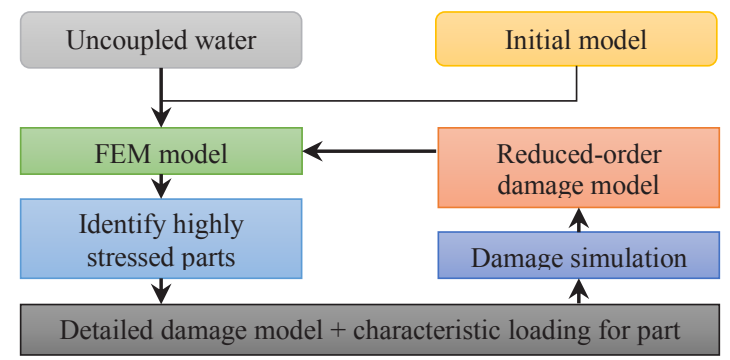

Fig. 7. Iterative process of model creation

The initial model is loaded by water loads from a rigid ditching simulation. The results are searched for locations that are highly stressed and may be damaged. For this locations a high granularity FEM model is created. For this damage FEM model, characteristic loading states are extracted from the fuselage FEM model. The damage simulation is carried out and an appropriate surrogate model is chosen that can reproduce the needed kinematics of the damage. Then an appropriate loading over deformation curve is extracted and the reduced order model is introduced into the fuselage FEM model. The procedure is then repeated with the new model as the occurrence of damage may change the loading of other parts in the model. If no new critical locations are found the iteration is stopped.

These steps need a high degree of manual interaction as it cannot easily be ascertained how big the detailed damage model should be to get realistic boundary constraints or which assumptions needs to be made to get the characteristic loading. If again the frame damage is considered the simulation of the frame together with clips and skin as shown in Fig. 5a is used instead of simulating just the frame as the kinematic boundary conditions are more realistic. As a surrogate model a hinge can be chosen in this example what again needs to be decided manually. This high degree of interaction is not problematic for an aircraft fuselage as the structure is very regular and only few different case needs to be minded. In [10] possible ideas for most relevant structural parts of a fuselage are already presented.

\section{Conclusions and Outlook}

In the present study we have presented a numerical framework that can be used for the coupled high-fidelity simulation of ditching problems in full scale. The partitioning of the computational domain was done by using the standard Dirichlet-Neumann approach. It is shown that this framework works well for cases with rigid and deformable structures if an implicit structural solver is used in combination with the proposed implicit coupling procedure. However, for the deformable validation case, the usage of an explicit structural solver as well as explicit coupling procedure lead to an unstable solution. Those results indicate that carefully designed coupling procedures need to be developed to facilitate the usage of explicit structural solvers for the partitioned FSI simulation of strongly coupled problems. This may require approaches other than standard Dirichlet-Neumann boundary conditions such as Robin boundary conditions or the Nitsche method (see e.g. [11]). Also, sub-cycling of the structural solver is advantageous if an implicit fluid solver is used.

It is shown that a structural model that on the one hand can capture the overall dynamics of the aircraft and on the other can directly simulate structural damage is not feasible. As explicit time integration is needed due to the high nonlinearities, a local refinement of damage 
relevant locations would lead to unfeasible short time steps and lead to an overall very poor computational efficiency. A method is presented how the highly localized damage behaviour of aeronautical structures can be incorporated in full size aircraft models of a granularity that can be handled by today's computers. This is done by reduced order models that account for the kinematic effects of structural damage. An iterative process is proposed how locations can be identified where this damage models should be placed in the aircraft fuselage.

\section{Acknowledgements}

The research leading to these results has received funding from the European Union's Horizon 2020 research and innovation programme under grant agreement No 724139.

\section{References}

1. Haupt, M.; Niesner, R.; Unger, R.; Horst, P. Computational Aero-Structural Coupling for Hypersonic Applications. Proceedings of the 9th AIAA/ASME Joint Thermophysics and Heat Transfer Conference, San Francisco, United States, 2006.

2. Fernández, M.; Mullaert, J. Convergence and Error Analysis for a Class of Splitting Schemes in Incompressible Fluid-Structure Interaction. IMA Journal of Numerical Analysis 2015, Volume 36, Issue 4, Pages 1748-1782, DOI: 10.1093/imanum/drv055.

3. Iafrati, A.; Grizzi, S.; Siemann, M.; Benítez Montañés, L. High-Speed Ditching of a Flat Plate: Experimental Data and Uncertainty Assessment. Journal of Fluids and Structures 2015, Volume 55, Pages 501-525, DOI: 10.1016/j.jfluidstructs.2015.03.019.

4. Reinhard, M.; Korobkin, A.; Cooker, M. Water Entry of a Flat Elastic Plate at High Horizontal Speed. Journal of Fluid Mechanics 2013, Volume 724, Pages 123-153, DOI: 10.1017/jfm.2013.155.

5. Niesner, R.; Dannemann, F.; Haupt, M.; Horst, P.; Schreiber, A. An MpCCI-based Software Integration Environment for Hypersonic Fluid-Structure Problems. Proceedings of the 7th MpCCI User Forum, Sankt Augustin, Germany, 2006.

6. Causin, P.; Gerbeau, J.; Nobile, F. Added-Mass Effect in the Design of Partitioned Algorithms for Fluid--Structure Problems. Computer methods in applied mechanics and engineering 2005, Volume 194, Issue 42-44, Pages 4506-4527, DOI: 10.1016/j.cma.2004.12.005.

7. Greenhow, M.; WM, L. Nonlinear-Free Surface Effects: Experiments and Theory. 1983, Cambridge: Massachusetts Institute of Technology, Department of Ocean Engineering.

8. Arai, M.; Miyauchi, T. Numerical Simulation of the Water Impact on Cylindrical Shells Considering Fluid-Structure Interaction. Journal of the Society of Naval Architects of Japan 1997, Volume 1997, Issue 182, Pages 827-835.

9. Ionina, M.; Korobkin, A. Water Impact on Cylindrical Shell. Proceedings of the 14th Int. Workshop on Water Waves and Floating Bodies, Michigan, United States, 1999.

10. Waimer, M.; Kohlgrüber, D.; Hachenberg, D.; Voggenreiter, H. The Kinematics Model - A Numerical Method for Development of a Crashworthy Composite Fuselage Design of Transport Aircraft. Proceedings of the 6th Triennial International Aircraft Fire and Cabin Safety Research Conference, Atlantic City, United States, 2010.

Burman, E.; Fernández, M. Explicit Strategies for Incompressible Fluid-Structure Interaction Problems: Nitsche Type Mortaring Versus Robin--Robin Coupling. International Journal for Numerical Methods in Engineering 2014, Volume 97, Issue 10, Pages 739-758, DOI: 10.1002/nme.4607. 